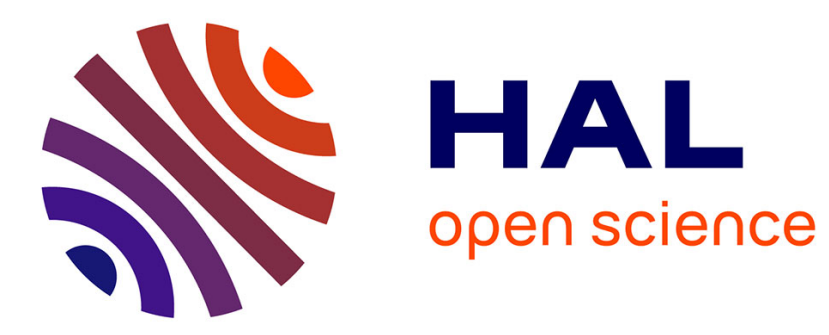

\title{
Transverse photodeflection signal formation in the framework of wave optics theory
}

\author{
A. Glazov, K. Muratikov
}

\section{To cite this version:}

A. Glazov, K. Muratikov. Transverse photodeflection signal formation in the framework of wave optics theory. Journal de Physique IV Proceedings, 1994, 04 (C7), pp.C7-71-C7-74. 10.1051/jp4:1994718 . jpa-00253241

\section{HAL Id: jpa-00253241 https://hal.science/jpa-00253241}

Submitted on 1 Jan 1994

HAL is a multi-disciplinary open access archive for the deposit and dissemination of scientific research documents, whether they are published or not. The documents may come from teaching and research institutions in France or abroad, or from public or private research centers.
L'archive ouverte pluridisciplinaire HAL, est destinée au dépôt et à la diffusion de documents scientifiques de niveau recherche, publiés ou non, émanant des établissements d'enseignement et de recherche français ou étrangers, des laboratoires publics ou privés. 


\title{
Transverse photodeflection signal formation in the framework of wave optics theory
}

\author{
A. Glazov and K. Muratikov
}

A.F. Ioffe Physicotechnical Institute of the Academy of Sciences of Russia, Politekhnicheskaya St. 26, 194021 St. Petersburg, Russia

\begin{abstract}
The calculation of the transverse photodeflection signal in the framework of wave optics proves to be a more accurate approach compared to that of geometrical optics. The influence of the account of wave optics effects on a signal behavior was theoretically analyzed under various conditions. It was shown that a difference between the signals calculated using both approaches, is most essential for a phase signal component.
\end{abstract}

\section{INTRODUCTION}

The transverse photodeflection (TPD) technique has become of special interest within the last few years [1-5] for the investigation of thermal properties of various objects. For instance, it allows determination of thermal diffusivity locally and without contact in a selected lateral (parallel to the sample surface) direction. Under some conditions, quantitative photodeflection investigation is a complicated procedure needing a numerical analysis of the TPD signal. Therefore, an accuracy of a measurement depends on the theoretical approach to the signal formation.

At present, the theory of TPD methods used for these purposes is based on the notions of geometric optics [6,7]. We showed elsewhere [8,9] for a normal photodeflection signal that this approximation, neglecting the diffraction of a probe laser beam on the thermal lens (TL) and the probe beam intensity distribution in the region of the $\mathrm{TL}$, can be responsible for a considerable difference between experimental and calculated data. This approach increases the accuracy of parameter determination, especially when phase data of the PD measurement are used.

Here we present the wave optics theory for the TPD signal formation and consider results of its comparison with the geometrical optics approach.

\section{WAVE OPTICS THEORY OF TPD SIGNAL FORMATION}

The wave optics approach to the TPD signal formation consists in the calculation of the probe laser beam intensity distribution at a photodetector plane after an interaction with the TL using the Fresnel-Kirchhof integral, and then the calculation of the TPD signal itself for a given photoreceiver. The latter consists of two photosensitive elements with a border, perpendicular to the surface of the object under study (Fig. 1). The TPD signal is then proportional to the difference between a probe beam intensity distribution at the photodetector after an interaction with the TL and that without the TL effect: 


$$
S(t)=2 \eta \int_{0}^{\infty} d y^{\prime} \int_{-\infty}^{\infty} d z^{\prime}\left(I\left(y^{\prime}, z^{\prime}\right)-I_{0}\left(y^{\prime}, z^{\prime}\right)\right)=2 \eta \int_{0}^{\infty} d y^{\prime} \int_{-\infty}^{\infty} d z^{\prime}\left(U_{0}\left(y^{\prime}, z^{\prime}\right) \delta U\left(y^{\prime}, z^{\prime}, t\right)+\text { compl.conj }\right)
$$

where $\eta$ is the proportionality coefficient between the light intensity at the photodetector and the electric output; $U_{0}\left(y^{\prime}, z^{\prime}\right)$ is the complex amplitude of the probe laser beam in the absence of the TL; $U\left(y^{\prime}, z^{\prime}, t\right)$ describes the change of the amplitude due to the interaction of the probe beam with the $T L$. $U\left(y^{\prime}, z^{\prime}, t\right)$ can be found by using the Fresnel-Kirchhof integral and the fact that the effect of the TL on the probe beam is very small and the change of the probe beam amplitude just after the interaction with the TL is

$$
\delta U(y, z, t) \cong U_{0}(y, z, t) i \varphi(y, z, t),
$$

where

$$
\varphi(y, z, t)=\frac{2 \pi}{\lambda} \cdot \frac{d n}{d T} \int_{-\infty}^{\infty} T(x, y, z, t) d x
$$

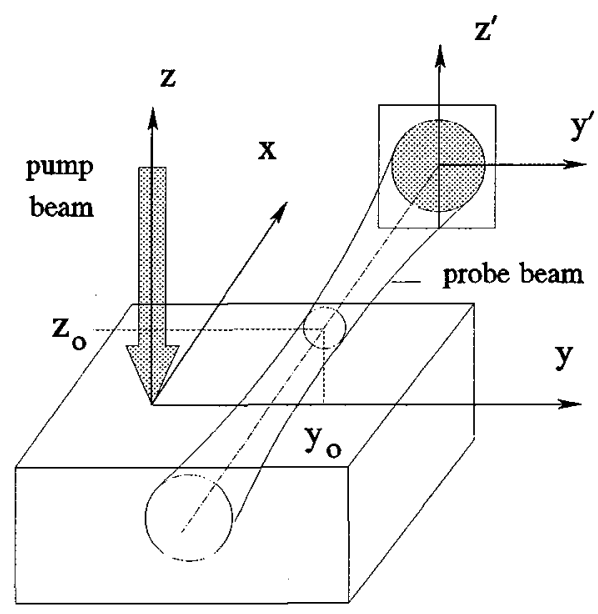

Fig.1. The geometry of the TPD technique.

is the change of the optical phase due to passing through the TL; $\lambda$ is the wavelength of the probe beam; $\mathrm{dn} / \mathrm{dT}$ is the derivative of the medium refractive index, and $\mathrm{T}$ is the non-stationary component of the medium temperature. Thus, assuming Gaussian distribution of the electromagnetic field of the laser probe beam without the action of the TL in the form

$$
U_{0}(y, z)=\frac{\sqrt{I_{0}} i k r}{\sqrt{\pi}\left(x+i k r^{2}\right)} \exp \left(-\frac{\left(y-y_{0}\right)^{2}+\left(z-z_{0}\right)^{2}}{2 r^{2}\left(1+i x / k r^{2}\right)}\right)
$$

one can integrate (1) over the y' and z' and obtain for the time-Fourier transform of the TPD signal

$$
\begin{gathered}
S(\omega)=\frac{2 I_{0} \eta}{\sqrt{\pi} \lambda r} \cdot \frac{\partial n}{\partial T} \int_{-\infty}^{\infty} d y \int_{-\infty}^{\infty} d k_{y} T\left(0, k_{y}, \omega\right) \exp \left(i k_{y} y-\gamma_{g} z_{0}+\frac{\left(\gamma_{g} r\right)^{2}}{4}-\frac{\left(y-y_{0}\right)^{2}}{r^{2}}\right) \\
\times i \operatorname{erf}\left(i \frac{y-y_{0}}{\sqrt{2} r}\right)\left[1+\operatorname{erf}\left(-\frac{\gamma_{g} r}{2}+\frac{z_{0}}{r}\right)\right],
\end{gathered}
$$

where $x$ is the distance from the focal plane; $k=2 \pi / \lambda ; r$ is the probe beam radius at the focus; $T\left(k_{x}, k_{y}, \omega\right)$ is the Fourier transform of the non-stationary component of the object surface temperature $\mathrm{T}(\mathrm{x}, \mathrm{y}, \mathrm{z}=0, \mathrm{t})$ over the coordinates $\mathrm{x}$ and $\mathrm{y}$ and time; $\gamma_{\mathrm{g}}=\left(\mathrm{k}_{\mathrm{y}}+\mathrm{i} \omega / \kappa_{\mathrm{g}}\right)^{1 / 2} ; \kappa_{\mathrm{g}}$ is the thermal diffusivity of the medium near the object. So, expression (5) represents the TPD signal calculated within the framework of wave optics when the finite radius of the probe beam is taken into account and a focal plane of the probe beam coincides with the center of the TL. The latter is assumed to be thinner than a probe beam waist length. 


\section{COMPARISON OF RESULTS OF WAVE AND GEOMETRIC APPROACHES TO THE TPD SIGNAL CALCULATION}

For a detailed analysis, computations of the TPD signal for a homogeneous semi-infinite sample were made using three approximations, namely the wave optics one, the geometrical optics one, and an approximation with an infinitesimally small probe beam radius. In the latter case, expressions for the wave and geometrical optics are same. We consider here only the case of homogeneous objects. The harmonic law for a description of a pump beam modulation was assumed.

First of all, we study the dependence of the TPD signal on the modulation frequency.

Figure 2 shows the TPD phase dependencies on the square root of the frequency at and $y_{0}=3 \mathrm{r}$ respectively for two probe beam radii $\mathrm{r}=35 \mu \mathrm{m}$ and $r=50 \mu \mathrm{m}$. The vertical offset $z_{0}$ was chosen equal to $2 \mathrm{r}$ for all cases as a compromise between minimizing this offset and the reduction of the probe beam diffraction on the sample. The pump beam radius was chosen as small as $1 \mu \mathrm{m}$.

The thermal diffusivity of the deflection medium was $0.2 \mathrm{~cm}^{2} / \mathrm{s}$. The difference between the phase calculated within the framework of wave $\left(\varphi_{w}\right)$ and geometrical optics $\left(\varphi_{\mathrm{w}}\right)$ takes place already at some hundred $\mathrm{Hz}$ and increases with the modulation frequency and the probe beam radius. At modulation frequencies higher than $10 \mathrm{kHz}$ the phase difference is about several tens of degrees. It should be noted, that at a small $y_{0}$ the

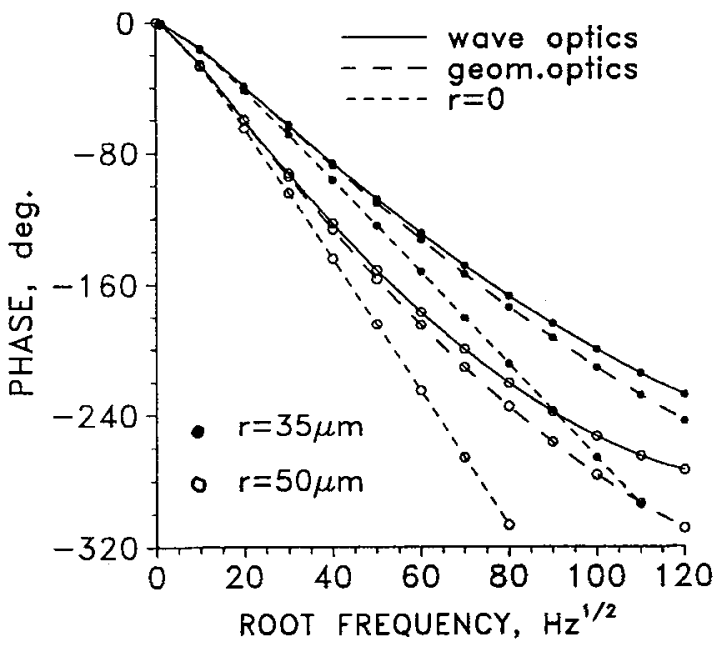

Fig.2. Frequency dependence of the TPD signal phase. wave optics signal phase is smaller than that of the geometrical optics signal, while at a large $y_{0}$ the relation is opposite. The amplitude behavior is noticeably different only at $y_{0}$ less than the probe beam radius. This is important when the dependence of the TPD phase on the lateral offset is used for the thermal diffusivity determination.

Let us consider the behavior of the TPD signal as a function of the lateral offset $y_{0}$. Figures 3 present these dependencies at the modulation frequency of $100 \mathrm{~Hz}$ for $\kappa_{0}=1 \mathrm{~cm}^{2} / \mathrm{s}$ and at the modulation frequency of $3000 \mathrm{~Hz}$ for $\kappa_{0}=0.001 \mathrm{~cm}^{2} / \mathrm{s}$. In the former case, the geometrical and wave approach lead to the same results for the TPD phase. The difference from the case of $r=0$ is about one degree. In the second case, the difference changes itself from -3 degrees at $y_{0} \approx 0 \mu \mathrm{m}$ to +10 degrees at $\mathrm{y}_{0}=300 \mu \mathrm{m}$, whereas the account of a finite probe beam radius results in the shift about 27 degrees. The amplitude behavior within the framework of geometrical theory is nearly independent on the probe beam radius. The amplitude calculated by wave theory is shifted to the side of large $y_{0}$. An analysis shows that a difference between the two approaches increases with the ratio $\mathrm{r} / \mu_{\mathrm{eff}}$, where $\mu_{\text {eff }}$ is the effective thermal wave length near the probe beam. At a large thermal diffusivity of a sample, $\mu_{\text {eff }} \approx \mu_{\text {sample }}$.

\section{CONCLUSIONS}

Thus, the use of the conventional approach for the formation of the transverse photodeflection signal in the framework of geometrical optics leads often to an incorrect interpretation of experimental data. Wave optics effects begin to play an important role when the thermal diffusion length of a sample or a deflecting medium is smaller than approximately half the probe beam radius. Wave optics effects appear 

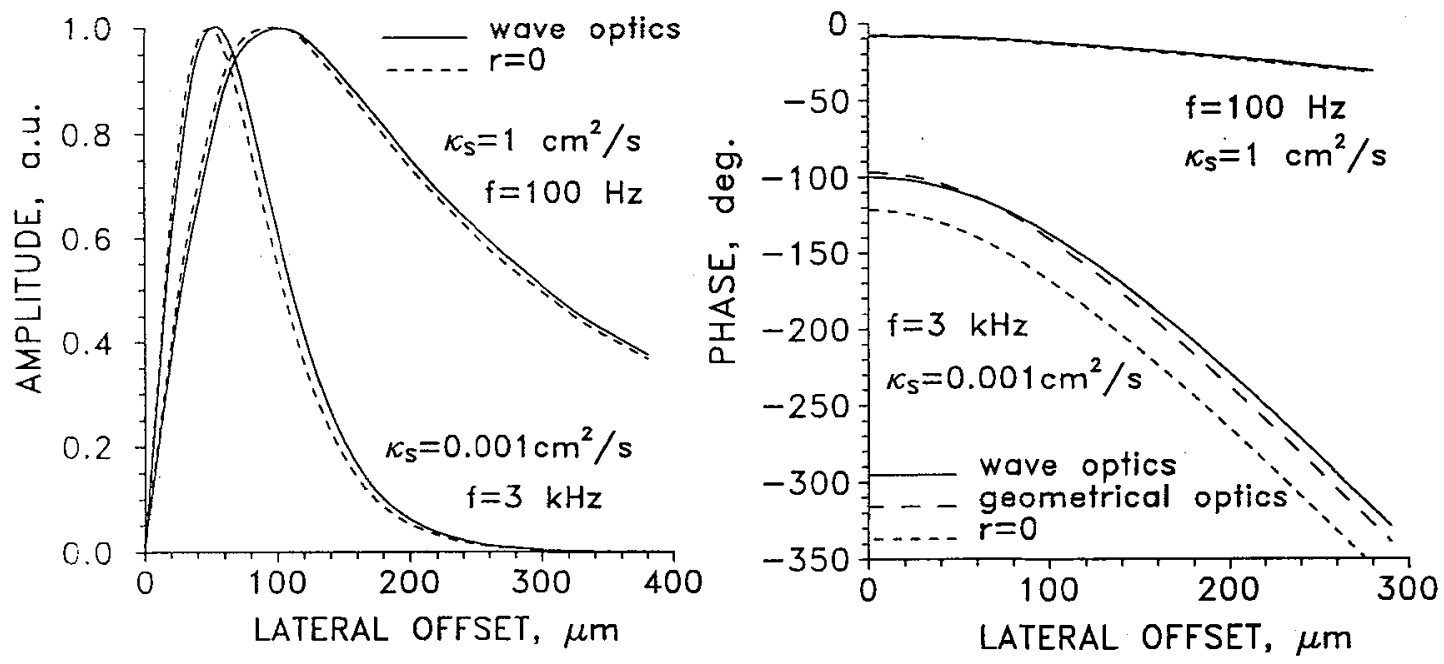

Fig. 3. The TPD signal amplitude and phase vs lateral offset $y_{0}$ at $r=45 \mu \mathrm{m}, \mathrm{z}_{0}=100 \mu \mathrm{m}$, and at the heating beam radius of $5 \mu \mathrm{m}$.

first in the phase component of the signal.

A wave optics approach to the formation of the transverse photodeflection signal has to be used when the thermal diffusivity of a sample is small compared to that of the deflecting medium, at a high frequency, or at a large probe beam radius.

\section{ACKNOWLEDGMENTS}

The support of this research by the Alexander von Humboldt Foundation of Germany is gratefully acknowledged. The authors would also like to thank Dr. H.-G. Walther for the fruitful discussion.

\section{References}

[1] Kuo P.K., Sendler E.D., Favro L.D., Thomas R.L., Kim D.S., Zhang Shu-yi, Inglehart L.J., Fournier D.,Boccara A.C., and Yacouby N.,Can.J.Phys. 64,(1986) 1165-1167.

[2] Salazar A., Sanchez-Lavega A., and Fernandez J.,J.Appl.Phys.69,(1991) 1216-1223.

[3] Figari A.,J.Appl.Phys,71,(1992) 3138-3142.

[4] Kantala J., Jaarinen J., and Kuo P.K.,Appl.Phys A 55,(1992) 586-695.

[5] Rantala J., Wei Lanhua,Kuo P.K. Jaarinen J.,Luukkala M., and Thomas R.L., J. Appl. Phys,73, (1993) 2714-2723.

[6] ickson W.B.,Amer N.M.,Boccara A.C., and Fournier D., Appl. Optics 20,(1981) 1333-1344.

[7] Namodt L.C., and Murphy J.C., J.Appl.Phys,52,(1981) 4903-4914.

[8] Slazov A., and Muratikov K., Opt. comm. 84,(1991) 283-289.

[9] (jlazov A., and Muratikov K., Photoacoustic and Photothermal Phenomena III, Springer Series in (optical Sciences 69, (Springer, Berlin, 1991) pp.528-530. 